\title{
Precursores de la Competencia Lectora Inicial en Escolares Hispanoparlantes de Nivel Socioeconómico Vulnerable
}

\author{
Precursors of Early Reading Competence in \\ Spanish-Speaking Children from Vulnerable Backgrounds
}

\author{
Alejandra Balbi \\ Universidad Católica del Uruguay
}

\author{
Alexa von Hagen \\ Nanyang Technological University
}

\author{
Carola Ruiz y Ariel Cuadro \\ Universidad Católica del Uruguay
}

\begin{abstract}
La identificación de precursores de la competencia lectora inicial es una estrategia prometedora para prevenir dificultades lectoras. Sin embargo, existen dudas sobre la aplicabilidad de la evidencia disponible al desarrollo lector en ortografías superficiales, como el español, y al caso particular de niños de nivel socioeconómico (NSE) vulnerable. Para resolver esta duda y mediante análisis de regresión múltiple, se estimó el poder explicativo de las habilidades (a) vocabulario expresivo, (b) conciencia fonológica y (c) correspondencia entre fonemas y grafemas, evaluadas al inicio del primer año escolar, sobre la competencia lectora inicial a inicios del primer y segundo año escolar en una muestra intencional de 104 niños hispanoparlantes que asistían a 6 escuelas privadas de NSE vulnerable de Montevideo, Uruguay. Al inicio del primer año escolar, los 3 precursores lograron explicar el 58\% de la varianza de la competencia lectora inicial. Sin embargo, un año después, al inicio del segundo año escolar, estos mismos precursores solo lograron explicar un $12 \%$ de las diferencias individuales en la competencia lectora inicial. Estos hallazgos contribuyen a una base empírica para la identificación de precursores de la competencia lectora inicial en niños hispanoparlantes de NSE vulnerable.
\end{abstract}

Palabras clave: precursores, alfabetización, lectura inicial, nivel socioeconómico, español

\begin{abstract}
The identification of precursors of early reading competence is a promising strategy to prevent reading difficulties. However, it remains unclear whether the available evidence is applicable to reading development in superficial orthographies, such as Spanish, and specifically to population of children from vulnerable backgrounds. To answer this question, multiple regression analysis was used to estimate the explanatory power of (a) expressive vocabulary, (b) phonological awareness, and (c) phoneme-grapheme correspondence skills-measured at the beginning of the first year of primary school-regarding the early reading competence of a purposive sample of 104 first and second grade Spanish-speaking children attending 6 vulnerable private schools in Montevideo, Uruguay. At the beginning of the first year of primary school, these 3 precursors explained $58 \%$ of the variance identified in early reading competence. However, one year later, at the beginning of second year of primary school, the same precursors only explained $12 \%$ of individual differences in early reading competence. These findings contribute to an empirical body of evidence for the identification of precursors of early reading competence in Spanish speaking children from vulnerable backgrounds.
\end{abstract}

Keywords: precursors, literacy, reading, socioeconomic status, Spanish

El desarrollo de la competencia lectora es uno de los logros más importantes que un niño experimenta al ingresar a la escuela primaria. A pesar de los esfuerzos realizados por maestros y padres, muchos niños tienen dificultades para alcanzar este objetivo. Una estrategia promisoria para abordar este problema es el monitoreo temprano de habilidades lingüísticas sobre las cuales se construye el aprendizaje inicial de la lectura. La identificación de estas habilidades previas, comúnmente llamadas precursores de la adquisición de la lectura, permite distinguir de forma temprana entre estudiantes que probablemente aprenderán a leer

Alejandra Balbi, Departamento de Psicología del Desarrollo y Educacional, Universidad Católica del Uruguay, Montevideo, Uruguay; Carola Ruiz y Ariel Cuadro, Departamento de Neurocognición, Universidad Católica del Uruguay, Montevideo, Uruguay; Alexa von Hagen, National Institute of Education, Nanyang Technological University, Singapore.

Esta investigación se llevó a cabo gracias al apoyo financiero de las fundaciones Niños con Alas y Fe y Alegría. Los autores declaran la ausencia de conflicto de intereses.

La correspondencia relativa a este artículo debe ser dirigida a Alejandra Balbi, Departamento de Psicología del Desarrollo y Educacional, Universidad Católica del Uruguay, Av. 8 de Octubre 2801, Montevideo, CP 11600, Uruguay. E-mail: abalbi@ucu.edu.uy 
con la instrucción regular ofrecida en el aula y aquellos que puedan necesitar apoyo adicional para aprender a leer (Scarborough, 2009).

La identificación de precursores de la competencia lectora inicial ofrece numerosas implicaciones prácticas para orientar a docentes y especialistas involucrados en la enseñanza de la lectura. Sin embargo, muchos de los aportes en esta línea de investigación provienen de estudios realizados con poblaciones angloparlantes. El sistema de escritura del inglés presenta numerosas diferencias con el español, así como también con otros sistemas de escritura, que se caracteriza por tener relaciones predominantemente biunívocas entre grafemas y fonemas (para una revisión de las características atípicas del inglés en relación a otros sistemas de escritura alfabéticos ver Share, 2008). De esta forma, es cuestionable si la amplia evidencia científica proveniente de investigaciones con poblaciones angloparlantes también puede ser empleada para orientar implicancias educativas en poblaciones que aprenden a leer en español.

Más allá de las diferencias entre los sistemas de escritura del español y el inglés, una preocupación especialmente relevante en muchos países hispanoparlantes es la situación de escolares de nivel socioeconómico (NSE) vulnerable. Existen amplios datos que indican que esta población presenta un riesgo elevado de experimentar dificultades en el aprendizaje de la lectura al iniciar su escolaridad (Arriaga, Fenson, Cronan \& Pethick, 1998; Cuadro, Barg, Navarrete \& Suero, 2008; Hart \& Risley, 1995; Huttenlocher, Waterfall, Vasilyeva, Vevea \& Hedges, 2010). La disponibilidad de recursos económicos y de gestión humana para apoyar el desarrollo de la competencia lectora en contextos de NSE vulnerable es extremadamente limitada y los retos cotidianos son múltiples y diversos. Por lo tanto, contar con herramientas basadas en la evidencia resulta fundamental para distribuir de forma eficiente apoyos especializados y atender a los escolares que tengan mayores probabilidades de presentar dificultades en el desarrollo de la competencia lectora inicial.

En este marco, nuestro estudio se propuso contribuir a la construcción de una base empírica que pueda servir de orientación para la identificación de precursores de la competencia lectora inicial en escolares hispanoparlantes de NSE vulnerable. Más específicamente, evaluamos el poder explicativo de las habilidades de (a) vocabulario expresivo, (b) conciencia fonológica y (c) correspondencia entre fonemas y grafemas sobre la competencia lectora inicial de escolares hispanoparlantes de NSE vulnerable. Para ello, recolectamos medidas sobre estos tres precursores al inicio del primer año escolar y analizamos hasta qué punto estas variables son capaces de explicar la variabilidad observada en el desarrollo de la competencia lectora medida al inicio del primer y segundo año escolar.

\section{Identificación Temprana de Precursores de la Competencia Lectora Inicial}

En muchos contextos educativos la identificación de dificultades en el desarrollo de la competencia lectora se rige por una espera pasiva hasta que se alcanza una discrepancia de al menos dos años entre el nivel de lectura esperado de acuerdo a la edad o el grado escolar del alumno y el nivel de lectura que realmente es alcanzado (Kavale, 2005). Esta forma de proceder, que algunos autores llaman esperar el fracaso (Reschly, 2005), conlleva numerosas consecuencias negativas (Tunmer \& Greaney, 2010). En el momento en el cual esta discrepancia se hace evidente, es probable que el retraso en la lectura se haya consolidado como una dificultad severa. Por consiguiente, el efecto de intervenciones educativas en muchos casos llega demasiado tarde para revertir las dificultades (Carta et al., 2014). Con el paso del tiempo, no solo se agrava la dificultad lectora, sino que también aumenta la probabilidad de consolidación de un fracaso académico, que en muchos casos trasciende el ámbito cognitivo y conlleva severas consecuencias emocionales (Boyes, Leitao, Claessen, Badcock \& Nayton, 2016).

Afortunadamente, estas prácticas educativas tradicionales comienzan a ser cuestionadas y sustituidas por enfoques preventivos centrados en la identificación temprana de dificultades en la lectura. El monitoreo temprano de precursores de la competencia lectora inicial juega un rol primordial en este sentido (Lam \& Mcmaster, 2014). Al identificar alumnos que presentan dificultades en estas habilidades, es posible proporcionar intervenciones preventivas y mitigar una gran mayoría de las dificultades en la competencia lectora inicial (Vellutino, Scanlon, Zhang \& Schatschneider, 2008). Esto es un aporte importante, ya que las prácticas educativas basadas en la identificación temprana de dificultades lectoras parecerían ser más eficaces y económicas a largo plazo.

Son varias las habilidades cognitivas que han sido estudiadas en los enfoques preventivos que hemos nombrado anteriormente para identificar alumnos que tienen mayor riesgo de presentar dificultades en la 
lectura (Lam \& Mcmaster, 2014). Entre ellas se encuentran diversas habilidades de lenguaje oral (e.g., conciencia fonológica, vocabulario), conocimientos iniciales sobre el sistema de escritura (e.g., correspondencias entre fonemas y grafemas) y el funcionamiento intelectual general, entre otras (Bridges \& Catts, 2011; Caravolas et al., 2012; Caravolas, Violín \& Hulme, 2005; Cardoso-Martins, 1995; Fricke, Szczerbinski, Fox-Boyer \& Stackhouse, 2016; Georgiou, Parrila \& Papadopoulos, 2008; Mayer \& Motsch, 2015; Roth, Speece \& Cooper, 2002; Stage, Abbott, Jenkins \& Berninger, 2003; Ziegler et al., 2010; Ziegler \& Ferrand, 1998).

Si bien en esta línea existen cada vez más publicaciones sobre estudios llevados a cabo con poblaciones hispanoparlantes, la gran mayoría de la evidencia disponible sigue estando basada en investigaciones con escolares angloparlantes. La construcción de una base empírica, que pueda guiar la identificación de precursores de la competencia lectora inicial en escolares hispanoparlantes de NSE vulnerable, lejos de representar un cuerpo de conocimientos acabado, se encuentra en pleno desarrollo. En este contexto, en nuestro estudio nos propusimos profundizar en el rol de los siguientes tres precursores de la competencia lectora inicial en escolares hispanoparlantes de NSE vulnerable: (a) vocabulario expresivo, (b) conciencia fonológica y (c) habilidades de correspondencia entre fonemas y grafemas. A continuación, profundizamos en el rol de cada una de estas variables en las habilidades de lectura inicial de escolares hispanoparlantes de NSE vulnerable.

\section{Vocabulario Expresivo y Competencia Lectora Inicial}

Existe amplia evidencia que vincula las habilidades de vocabulario expresivo con la competencia lectora inicial (Duff \& Hulme, 2012; Duff, Reen, Plunkett \& Nation, 2015; Harlaar, Hayiou-Thomas, Dale \& Plomin, 2008; Hart \& Risley, 1995; Muter, Hulme, Snowling \& Stevenson, 2004; Nation \& Cocksey, 2009; Nation \& Snowling, 2004; NICHD Early Child Care Research Network, 2005). Esta relación está basada en la idea de que los niños son capaces de producir numerosas palabras a nivel oral antes de encontrarse por primera vez con la presentación escrita de estas mismas palabras. De esta forma, conocimientos previos sobre la fonología de una palabra parecerían facilitar el acercamiento a la representación escrita de la misma durante las primeras etapas del aprendizaje lector (Lesglod \& Perfetti, 1981; Share, 1995). Por ejemplo, en un estudio reciente Wegener et al. (2018) reportaron que un grupo de niños angloparlantes de 9 y 10 años alcanzaron un mejor rendimiento al leer un conjunto de pseudopalabras que habían aprendido a producir a nivel oral en un entrenamiento previo, en comparación con un set de pseudopalabras con las que entraban en contacto por primera vez.

Específicamente en el contexto latinoamericano, Escobar y Meneses (2014) aportan datos relevantes a partir de un estudio en Chile con escolares hispanoparlantes. Sus resultados muestran que el nivel de vocabulario medido a inicios del primer año escolar mostró un rol predictivo de la lectura de palabras a finales del primer año escolar en niños de NSE vulnerable, pero no así en niños de NSE medio y alto. Aunque estos datos están basados en una medida de vocabulario receptivo (identificación de una imagen a partir de una palabra presentada a nivel oral), que no abarca las habilidades de vocabulario expresivo en las que nos centramos en este estudio, reflejan el déficit que muchos niños de NSE vulnerable presentan en esta habilidad lingüística, en comparación con niños de NSE medio y alto.

No solo se han descrito déficits en las habilidades de vocabulario de niños de NSE vulnerable, sino también en otras habilidades lingüísticas, como en la comprensión y producción de estructuras morfosintácticas y la comprensión y producción discursiva, entre otras (Hoff, 2013). Sin embargo, el déficit a nivel de las habilidades de vocabulario expresivo parece ser especialmente marcado desde una edad temprana. Así, por ejemplo, Arriaga et al. (1998) informaron que el tamaño del vocabulario expresivo del $80 \%$ de una muestra de niños entre 18 y 30 meses de edad de NSE vulnerable no superó valores equivalentes al percentil 50 de los valores normativos en niños de NSE medio y alto. También Hart y Risley (1995) indican que a la edad de 3 años los niños de NSE alto de su muestra emitían aproximadamente el doble de palabras que los participantes de NSE vulnerable.

\section{Conciencia Fonológica y Competencia Lectora Inicial}

Mientras que varios estudios han destacado el carácter universal de la conciencia fonológica en términos globales, como precursor de la competencia lectora inicial (Caravolas et al., 2012; Ziegler et al., 2010), existen dudas sobre las subhabilidades de conciencia fonológica que son necesarias para alcanzar una competencia 
lectora inicial en sistemas ortográficos de diferentes lenguas (Cardoso-Martins, 1995; Guardia, 2014; Mayer \& Motsch, 2015; Wimmer, Landerl, Linortner \& Hummer, 1991; Wimmer, Landerl \& Schneider, 1994; Ziegler et al., 2010).

Más específicamente, la profundidad ortográfica del sistema de escritura parece ser el moderador clave en este contexto (para una revisión detallada del concepto de profundidad ortográfica ver Schmalz, Marinus, Coltheart \& Castles, 2015). Este concepto se refiere al grado de complejidad de las relaciones entre los sonidos de una palabra y su representación escrita. Las así llamadas ortografías superficiales, como el español, se basan en correspondencias predominantemente biunívocas entre grafemas y fonemas. De esta forma, un lector principiante rápidamente es capaz de decodificar la gran mayoría de las palabras escritas, una vez que se apropia de las reglas de correspondencia entre grafemas y fonemas (Defior \& Serrano, 2014). Así, subhabilidades de conciencia fonológica centradas en la manipulación de fonemas aislados o agrupaciones de dos fonemas (e.g., en sílabas) son un precursor importante para aprender las correspondencias entre fonemas y grafemas (e.g., $/ \mathrm{m} / / \mathrm{e} / / \mathrm{s} / / \mathrm{a} /=<\mathrm{m}><\mathrm{e}><\mathrm{s}><\mathrm{a}>$ ) (Cardoso-Martins, 1995; Defior \& Serrano, 2014; Guardia, 2014; Wimmer et al., 1994).

Por el contrario, las denominadas ortografías profundas, como el inglés, se caracterizan por seguir correspondencias múltiples entre grafemas y fonemas, las que son más difíciles de aprender (Geva \& Siegel, 2000; Share, 2008). En muchos casos, estas correspondencias implican relaciones entre un fonema y varios grafemas (e.g., /u:/ = <ough> en / $\mathrm{ru}: /=<$ through > —en español a través—; Schmalz et al., 2015). Esto implica que en muchos casos las subhabilidades de conciencia fonológica centradas en la manipulación de unidades fonológicas mayores que el fonema, como la rima, jueguen un rol importante para aprender las correspondencias entre grafemas y fonemas necesarias para aprender a leer (Bryant, MacLean, Bradley \& Crossland, 1990; Cardoso-Martins, 1995; Guardia, 2014).

Esta diferencia entre el rol de las subhabilidades de conciencia fonológica precursoras de la competencia lectora inicial en sistemas de escritura superficiales y profundos, conlleva importantes consecuencias para la prevención e identificación temprana de dificultades en la competencia lectora inicial en niños hispanoparlantes. En coherencia con los contrastes entre sistemas de escritura superficiales y profundos que hemos descrito anteriormente, la evaluación de subhabilidades de conciencia fonológica centradas en la manipulación de fonemas o sílabas, pero no de unidades mayores, como la rima, parecería cobrar mayor relevancia. Los resultados de Cardoso-Martins (1995) en portugués y Wimmer et al. (1994) en alemán, dos lenguas con sistemas de escritura predominantemente superficiales, confirman esta idea. También en español, los resultados de Guardia (2014), en un estudio en Chile con niños de NSE vulnerable en edad preescolar, reflejan que la habilidad de identificar rimas no juega un rol en la explicación de la variabilidad observada en diferentes medidas de competencia lectora inicial realizadas al final del primer año escolar.

\section{Correspondencia entre Fonemas y Grafemas y Competencia Lectora Inicial}

Otro precursor de la competencia lectora inicial, que ha recibido mucha atención en investigaciones pasadas, es la habilidad de establecer correspondencia entre fonemas y grafemas (Byrne \& Fielding-Barnsley, 1989; Ehri, 1998; Escobar \& Meneses, 2014; Foulin, 2005; Hulme, Bowyer-Crane, Carroll, Duff \& Snowling, 2012; Levin, Shatil-Carmon \& Asif-Rave, 2006). Mientras las habilidades de vocabulario expresivo y de conciencia fonológica que hemos descrito en las secciones anteriores se centran en el procesamiento de información auditivo-verbal, la habilidad de corresponder fonemas y grafemas implica coordinar el procesamiento de información de tipo auditivo-verbal y visual-ortográfico (Friedmann \& Coltheart, 2018). De esta forma, representa una primera aproximación para establecer relaciones entre unidades de lenguaje oral y escrito (Ziegler \& Goswami, 2006).

Sin embargo, la cantidad de contacto con materiales escritos disponible parecería moderar el rol de las habilidades de correspondencia entre fonemas y grafemas para explicar variaciones en la competencia lectora inicial en los primeros años escolares. Por ejemplo, en un estudio de Escobar y Meneses (2014) este precursor, medido al inicio del primer año escolar por medio de una tarea de denominación de letras, jugó un rol significativo en predecir el rendimiento lector alcanzado por los participantes al final del primer año escolar solo en niños hispanoparlantes de NSE vulnerable, pero no de NSE medio y alto. Es posible que los niños de NSE medio y alto hayan tenido mayores oportunidades de practicar la denominación de letras que otros niños de NSE vulnerable, ya que se trata de una tarea comúnmente impulsada por muchos padres de NSE medio y alto (Griffin \& Morrison, 1997; Piasta \& Wagner, 2010), así como por docentes de educación preescolar. De 
esta forma, la tarea de denominación de letras podría haber resultado poco sensible para captar diferencias en las habilidades de correspondencia entre fonemas y grafemas en los participantes de NSE medio y alto.

Otra tarea que también ha sido señalada como un índice de la habilidad de corresponder fonemas y grafemas es la escritura inventada de palabras (invented spelling en inglés), en la cual los niños deben transcribir los grafemas correspondientes a un fonema, una sílaba o una palabra presentada oralmente (Cunningham \& Cunningham, 1992; Gentry, 2000; Lombardino, Bedford, Fortier, Carter \& Brandi, 1997; Ouellette \& Sénéchal, 2008, 2017). A diferencia de una tarea tradicional de escritura de palabras, en la escritura inventada de palabras se puntúa positivamente que la correspondencia entre cada fonema y grafema dentro del ítem presentado sea correcta, sin tomar en cuenta la escritura ortográficamente correcta de la palabra. Un rendimiento exitoso exige la habilidad de establecer una correspondencia entre una unidad auditivo-verbal y visual-ortográfica, al igual que en la tarea de denominación de letras. Sin embargo, la presentación de unidades auditivo-verbales mayores (sílabas y palabras) impone exigencias cognitivas adicionales vinculadas con habilidades de conciencia fonológica (específicamente, segmentación fonémica) y de memoria de trabajo verbal (Ouellette \& Sénéchal, 2008). Estas exigencias adicionales imponen un mayor grado de dificultad sobre esta tarea, en comparación con la tarea de denominación de letras. Por consiguiente, es posible que una evaluación de las habilidades de correspondencia entre fonemas y grafemas por medio de la escritura inventada de palabras permita detectar con mayor sensibilidad diferencias individuales en el dominio de esta habilidad, que por medio de la denominación de letras.

\section{Nuestro Estudio}

Nuestro estudio forma parte de un proyecto de evaluación e intervención de alfabetización más amplio que tuvo por objetivo estudiar el impacto de una intervención. Fue llevado a cabo por la Facultad de Psicología de la Universidad Católica del Uruguay de la ciudad de Montevideo, en conjunto con dos organizaciones no gubernamentales. En particular, con el presente estudio nos propusimos el objetivo de investigar la identificación de precursores del desarrollo de la competencia lectora inicial en escolares hispanoparlantes de NSE vulnerable. Centramos nuestra atención específicamente en el poder explicativo de los siguientes tres precursores: (a) vocabulario expresivo, (b) conciencia fonológica y (c) correspondencia entre fonemas y grafemas, sobre la competencia lectora inicial, evaluándolos durante el primero y segundo año de la etapa escolar. Nos preguntamos:

1. ¿Hasta qué punto los tres precursores logran explicar la variabilidad observada en la competencia lectora inicial al inicio del primer año escolar?

2. ¿Hasta qué punto estos mismos precursores contribuyen en la explicación de la varianza revelada en la competencia lectora inicial un año más tarde, al inicio del segundo año escolar?

\section{Método}

\section{Participantes}

Seis escuelas privadas de NSE vulnerable fueron elegidas de forma intencional para participar del proyecto. Por un lado, las escuelas se ubicaban en zonas geográficas de NSE bajo, de acuerdo con la clasificación del Instituto Nacional de Estadística del Uruguay (2012). Por otro lado, las escuelas que participaron en el proyecto mayor informaron que el importe máximo de cuota mensual abonado por los padres de los alumnos no superó los 20 US\$. De acuerdo a una base de datos sobre las cuotas mensuales exigidas por todas las escuelas privadas en Uruguay, el importe mensual requerido puede variar entre $0 \mathrm{y}$ 1.000 US $\$$. Por lo tanto, el importe mensual solicitado por las escuelas privadas que participaron en el proyecto se ubica en el límite inferior de este rango y puede ser tomado como un indicador de un NSE vulnerable.

Si bien algunas de las escuelas que participaron en este estudio ofrecen clases de inglés como segunda lengua, la gran mayoría de la jornada escolar toma lugar en español, siendo esta la lengua materna de la gran mayoría de los alumnos. En Uruguay, los niños suelen asistir a la educación preescolar a partir de los 4 años de edad y son expuestos a situaciones letradas en forma lúdica. Sin embargo, la instrucción sistemática de alfabetización comienza en el primer año de la escuela primaria. La instrucción en conciencia fonológica no integra el currículo oficial del preescolar ni de la educación primaria y tanto métodos sintéticos como analíticos de enseñanza de la lecto-escritura son comunes (Administración Nacional de Educación Pública, 2013). 
En una primera instancia de screening evaluamos y seleccionamos 107 niños que rindieron por encima del percentil 25 de la distribución de puntajes de la muestra, excluimos tres alumnos con diagnósticos formales de trastornos del desarrollo, discapacidades intelectuales o sensoriales, de acuerdo a los registros escolares de cada institución educativa. Por lo tanto, la muestra final consistió en 104 escolares (47 niños y 57 niñas; edad: $M=6,4, D E=0,3$ años). Estos alumnos fueron evaluados (a) al inicio del primer año de primaria y (b) un año más tarde, al inicio del segundo año de primaria. En el segundo momento de evaluación 18 niños habían dejado de asistir a las escuelas participantes y, por lo tanto, en esta segunda etapa contamos con los datos de 86 niños (38 niños y 48 niñas; edad: $M=7,5, D E=0,3$ años).

\section{Instrumentos}

Seleccionamos tareas e instrumentos para medir cada uno de los precursores de la competencia lectora inicial.

Screening inicial. Para evaluar las habilidades iniciales de lectura de la muestra completa original utilizamos la prueba Identificación de Letras y Palabras de la Batería III Woodcock-Muñoz (Muñoz-Sandoval, Woodcock, McGrew \& Mather, 2005). En esta prueba los niños leen en voz alta grafemas (e.g., i, o, s, b), sílabas y palabras de dificultad creciente (e.g., la, pan, de, tren). Se les presentó a los niños un máximo de 76 ítems con una regla de interrupción de seis errores consecutivos. La consistencia interna de la prueba, evaluada por alfa de Cronbach, es de $\alpha=0,95$, en base a una muestra de personas de habla hispana de Estados Unidos, España y diferentes países de América Latina (Schrank et al. 2005). Desafortunadamente, no existen datos locales para Uruguay.

\section{Precursores de la competencia lectora inicial.}

Vocabulario expresivo. Para evaluar las habilidades de vocabulario expresivo, consideramos una tarea de comprensión oral que involucra aspectos semánticos, sintácticos y léxicos relacionadas con la producción de palabras orales. Utilizamos la prueba Comprensión Oral de la Batería III Woodcock-Muñoz (MuñozSandoval et al., 2005). En esta tarea el evaluador lee en voz alta un texto breve, al que le falta una palabra (e.g., Los niños estudian en la...; El café es negro, la leche es...). La tarea del niño consiste en nombrar la palabra faltante, a partir de la información brindada en el texto. A los niños se les presentó un máximo de 34 ítems con una regla de interrupción de seis errores consecutivos. La consistencia interna de la prueba, evaluada por alfa de Cronbach, es de $a=0,93$ en base a una muestra de personas de habla hispana de Estados Unidos, España y diferentes países de América Latina (Schrank et al., 2005). Nuevamente, no contamos con datos locales para Uruguay.

Conciencia fonológica. Con respecto a la evaluación de las habilidades de conciencia fonológica, en base a la revisión de la literatura previa, seleccionamos una tarea que incluyera tanto ejercicios de manipulación de fonemas y pares de fonemas como de unidades fonológicas mayores, como la rima. Esto nos permitió corroborar cuáles de las subhabilidades de conciencia fonológica juegan un rol en la explicación de la variabilidad observada en la competencia lectora inicial de niños hispanoparlantes de NSE vulnerable. Optamos por la prueba Discernimiento de Sonidos de la Batería III Woodcock-Muñoz (Muñoz-Sandoval et al., 2005). Esta prueba consta de las siguientes cuatro tareas: (a) rima (ejemplo: ¿Qué rima con casa/coche?); (b) eliminación de sílabas o fonemas (e.g., Si a dedo le quito do queda...); (c) sustitución de sílabas o fonemas (e.g., Si a carta le quito $t$ queda...) y (d) inversión de sílabas o fonemas (e.g., Escucha los sonidos de sal, si los digo al revés queda...). En todas las tareas el niño debe crear una nueva palabra a partir de una palabra dada. Cada tarea contiene 10-18 ítems, con una regla de discontinuación de tres errores consecutivos. La consistencia interna, evaluada por alfa de Cronbach, es $a=0,94$ para la prueba completa (Schrank et al., 2005). También aquí no existen datos locales para Uruguay.

Correspondencia entre fonemas y grafemas. Como medida de las habilidades de correspondencia entre fonemas y grafemas utilizamos una tarea de escritura inventada de palabras. Si bien muchos estudios previos comúnmente emplean tareas como la denominación de letras para medir los conocimientos sobre las reglas de correspondencia entre fonemas y grafemas (e.g., Escobar \& Meneses, 2014), pensamos que nuestros participantes podrían alcanzar puntajes cercanos al techo en una prueba de este tipo. La razón de esto es que, si bien la alfabetización sistemática en la lectoescritura en Uruguay comienza en el primer año escolar, los alumnos entran en contacto con el lenguaje escrito en numerosas propuestas lúdicas durante los años de educación preescolar. Especialmente tareas de denominación de letras son muy comunes en este contexto. 
Por lo tanto, pensamos que una tarea de escritura inventada de palabras podría ser más sensible para captar variaciones en las habilidades de correspondencia entre fonemas y grafemas en los participantes de nuestro estudio.

Utilizamos la prueba Ortografía de la Batería III Woodcock-Muñoz (Muñoz-Sandoval et al., 2005). En esta prueba el niño debe escribir grafemas, sílabas y palabras de dificultad creciente, siguiendo el dictado del evaluador. Todas las respuestas con una conversión de fonemas en grafemas aceptable según las reglas de correspondencia del español usado en América Latina, sin tomar en cuenta la corrección ortográfica en el caso de los ítems de palabras, fueron consideradas correctas (e.g., mallo x mayo). Se presentó a los niños un máximo de 58 ítems con una regla de interrupción de seis errores consecutivos. La consistencia interna de esta prueba, evaluada por alfa de Cronbach, es de $a=0,93$ (Schrank et al., 2005). Al igual que en las subpruebas que hemos descrito anteriormente, no existen datos locales para Uruguay.

Competencia lectora inicial. Para evaluar la competencia lectora inicial, nos orientamos en modelos cognitivos que entienden la lectura como una interacción de procesos léxicos, sintácticos y semánticos, desde el inicio del aprendizaje lector (Dijkstra \& van Heuven, 2002; Rumelhart, 1975, Julio/1977, 1994; Strijkers, Bertrand \& Grainger, 2015). Por lo tanto, seleccionamos tareas de lectura de palabras y oraciones que estuvieran enmarcadas en un contexto semántico. En sistemas de ortografía superficiales, como el español, para muchos lectores principiantes el mayor obstáculo surge a la hora de automatizar todos los procesos nombrados anteriormente y alcanzar una adecuada fluidez lectora (Carrillo \& Alegría, 2009; Defior \& Serrano, 2014; Landerl, Wimmer \& Frith, 1997; Wimmer \& Mayringer, 2002). Por lo tanto, en el segundo momento de evaluación de la competencia lectora inicial, al inicio del segundo año escolar, empleamos una tarea que, además de involucrar aspectos léxicos, sintácticos y semánticos, también nos aportara información sobre la velocidad de procesamiento implicada en la competencia lectora inicial de los niños.

Momento de evaluación 1 - inicios del primer año de escuela. Usamos la prueba Comprensión de Textos de la Batería III Woodcock-Muñoz (Muñoz-Sandoval et al., 2005) como medida de la competencia lectora inicial al inicio del primer año escolar. En esta tarea, los niños deben leer palabras y oraciones breves y, en base a la comprensión de lo leído, seleccionar una imagen correspondiente entre varias opciones (e.g., casa grande y se muestran cuatro imágenes, una casa pequeña, la casa grande y dos árboles de distinto tipo; dos árboles y se muestran cinco imágenes donde solo una corresponde a la opción correcta). Se presentó a los niños un máximo de 47 ítems con un criterio de interrupción de seis errores consecutivos. La consistencia interna de esta subprueba, evaluada por alfa de Cronbach, es de $a=0,91$ (Schrank et al., 2005), pero no contamos con datos locales para Uruguay.

Momento de evaluación 2 - inicios del segundo año de escuela. En esta segunda etapa de evaluación empleamos el Test de Eficacia Lectora (TECLE; Cuadro et al., 2005). En cada ítem del test el niño debe leer una frase incompleta y seleccionar la palabra correcta entre cuatro distractores semánticos y ortográficos (ejemplo: Tu pelota es de color...rogo-roco-robo-rojo). El test consta de 64 ítems, que se presentan en un tiempo límite de 5 minutos. De esta forma, el puntaje final equivale a la cantidad de respuestas correctas dentro del tiempo límite y refleja tantos aspectos de la precisión, velocidad y comprensión lectora. Este instrumento se ha baremado en Montevideo, Uruguay, y cuenta con una confiabilidad test-retest de $r=0,88$ (Cuadro et al., 2005).

\section{Procedimiento}

Nuestro estudio fue aprobado por el Comité de Ética de la Facultad de Psicología de la Universidad Católica del Uruguay. Enviamos folletos informativos a todos los padres de los niños de primer año de primaria de las seis escuelas que participaron en el proyecto más amplio, en los que explicábamos el objetivo del proyecto y la participación de los niños en el mismo. Además, junto con los folletos informativo, enviamos consentimientos informados a los padres de los niños y una vez que recepcionamos las autorizaciones firmadas, procedimos a continuar con la investigación.

El estudio constó de tres momentos de evaluación. Primero, en una etapa de screening, evaluamos las habilidades iniciales de lectura de la muestra completa $(n=165)$, que participó del proyecto de investigación más amplio en el que se enmarcó nuestro estudio. Esta evaluación tuvo lugar en el primer mes del año escolar correspondiente al primer año escolar. A partir de los resultados de esta evaluación, excluimos a 61 alumnos que no cumplían con los criterios de inclusión que detallamos en el apartado Participantes. 
En segundo lugar, los restantes 104 alumnos participaron de un primer momento de evaluación, el que también llevamos a cabo al inicio del primer año escolar. En esta instancia recolectamos información sobre la competencia lectora inicial de los niños y sobre tres precursores de la competencia lectora inicial: habilidades de (a) vocabulario expresivo, (b) de conciencia fonológica y (c) de correspondencia entre fonemas y grafemas. Toda la información fue recolectada en una sesión de aplicación llevada a cabo en una habitación tranquila asignada por la escuela.

Finalmente, un año más tarde, en un segundo momento de evaluación, al inicio del segundo año escolar, medimos nuevamente la competencia lectora inicial de los participantes. Esta información fue recolectada en una sesión de aplicación grupal llevada a cabo en el aula. En esta instancia, 18 alumnos que habían participado del primer momento de evaluación ya no eran alumnos de las seis escuelas privadas. Por lo tanto, los datos recolectados en este segundo momento de evaluación se limitaron a 86 niños.

La aplicación de todas las pruebas fue realizada por 10 estudiantes avanzados de psicología, que habían sido especialmente capacitados por el primer autor de este artículo.

\section{Análisis de Datos}

Para abordar nuestras preguntas de investigación, analizamos la correlación lineal de Pearson de los tres precursores (vocabulario expresivo, conciencia fonológica y correspondencia entre fonemas y grafemas) con la competencia lectora inicial medida al inicio de primer año escolar y al inicio de segundo año escolar, respectivamente. En base a estos resultados, seleccionamos los precursores para realizar dos análisis de regresión múltiple separados.

Realizamos un primer análisis de regresión múltiple por etapas, con la competencia lectora inicial alcanzada al inicio del primer año escolar como variable dependiente y los siguientes tres pasos de variables independientes: (a) vocabulario expresivo, (b) subhabilidades de conciencia fonológica (cancelación, sustitución e inversión) y (c) habilidades de correspondencia entre fonemas y grafemas.

Luego, completamos un segundo análisis de regresión múltiple. Nuevamente ingresamos las tres habilidades precursoras como variables independientes en los siguientes tres pasos: (a) vocabulario expresivo, (b) subhabilidades de la conciencia fonológica (cancelación e inversión) y (c) habilidades de correspondencia entre fonemas y grafemas. Como variable dependiente en esta instancia ingresamos la competencia lectora inicial alcanzada al inicio del segundo año escolar.

\section{Resultados}

En primer lugar, en la Tabla 1 presentamos los estadísticos descriptivos de todas las competencias que evaluamos. Luego, en la Tabla 2 presentamos los coeficientes de correlación de Pearson entre los precursores y la competencia lectora inicial, en ambos momentos de la medición. Por último, en la Tabla 3 mostramos los resultados del análisis de regresión para abordar nuestras preguntas de investigación.

La inclusión de las tres variables independientes mejoró el modelo y en total logró explicar el 58\% de la varianza de la competencia lectora inicial alcanzada al inicio de primer año, $R^{2}=0,58, F(1,98)=59,5$, $p<0,001$. Las habilidades de vocabulario expresivo mostraron una contribución independiente con un poder explicativo del $12 \%, R^{2}=0,12, F(1,102)=13,5, p<0,001$. Si bien las habilidades de conciencia fonológica lograron explicar el $21 \%$ de la varianza, $R^{2}=0,21, F(3,99)=10,5, p<0,001$, esta contribución se debió exclusivamente al aporte de la subhabilidad de inversión de fonemas y sílabas. Ni la tarea de cancelación ni la de sustitución de fonemas y sílabas contribuyeron a explicar la competencia lectora inicial alcanzada en esta etapa escolar. Por último, las habilidades de correspondencia entre fonemas y grafemas revelaron la mayor contribución independiente, explicando el $25 \%$ de la varianza de la competencia lectora inicial alcanzada al inicio de primer año escolar, $R^{2}=0,25, F(1,98)=59,4, p<0,001$. 
Tabla 1

Estadísticos Descriptivos de las Competencias Evaluadas

\begin{tabular}{|c|c|c|c|c|}
\hline Medida & $M$ & $D E$ & Min & Máx \\
\hline Vocabulario expresivo & 15,05 & 3,13 & 9,00 & 22,00 \\
\hline CF Rimas & 4,44 & 2,14 & 1,00 & 13,00 \\
\hline CF Cancelación & 2,35 & 1,71 & 0,00 & 6,00 \\
\hline CF Sustitución & 1,50 & 1,75 & 0,00 & 11,00 \\
\hline CF Inversión & 2,87 & 2,55 & 0,00 & 11,00 \\
\hline Correspondencia entre fonemas y grafemas & 19,93 & 610 & 8,00 & 37,00 \\
\hline Inicio del $1^{\mathrm{er}}$ año - Competencia lectora inicial & 7,60 & 5,42 & 4,00 & 29,00 \\
\hline Inicio del $2^{\circ}$ año $o^{\mathrm{a}}$ Competencia lectora inicial & 11,22 & 4,30 & 3,00 & 22,00 \\
\hline
\end{tabular}

Nota. $\mathrm{CF}=$ Conciencia fonológica. a $\mathrm{Al}$ inicio de $1 \mathrm{er}$ año contamos con los datos de $n=104$ niños; al inicio de $2^{\circ}$ solamente participaron 86 niños. Competencia lectora Inicial de $1^{\text {er }}$ año = Identificación de letras y palabras; Competencia lectora inicial de $2^{\circ}$ año $=$ TECLE.

Tabla 2

Correlaciones entre Precursores y Competencia Lectora Inicial al Inicio del $1^{\text {er }}$ y $2^{\circ}$ Año Escolar

\begin{tabular}{lcc}
\hline \multicolumn{1}{c}{ Precursor } & $\begin{array}{c}\text { Competencia lectora inicial } \\
\text { al inicio del 1er año escolar }\end{array}$ & $\begin{array}{c}\text { Competencia lectora inicial } \\
\text { al inicio de } 2^{\circ} \text { año escolar }\end{array}$ \\
\hline Vocabulario expresivo & $0,327^{* *}$ & $0,219^{*}$ \\
CF Rimas & 0,142 & 0,080 \\
CF Cancelación & $0,427^{* *}$ & $0,235^{*}$ \\
CF Sustitución & $0,349^{* *}$ & 0,108 \\
CF Inversión & $0,509^{* *}$ & $0,248^{*}$ \\
Correspondencia entre fonemas y grafemas & $0,698^{* *}$ & $0,302^{*}$ \\
\hline
\end{tabular}

Nota. $\mathrm{CF}=$ Conciencia fonológica. ${ }^{*} p<0,05,{ }^{* *} p<0,001$

Tabla 3

Regresión Múltiple por Pasos para Explicar la Varianza de la Competencia Lectora Inicial Alcanzada al Inicio del $1^{\text {er }}$ y $2^{\circ}$ Año Escolar

\begin{tabular}{|c|c|c|c|c|c|c|c|c|c|c|}
\hline \multirow{2}{*}{ Paso y precursor } & \multicolumn{5}{|c|}{$\begin{array}{l}\text { Competencia lectora inicial al inicio del } 1^{\text {er }} \\
\text { año escolar }\end{array}$} & \multicolumn{5}{|c|}{$\begin{array}{c}\text { Competencia lectora inicial al inicio del } 2^{\circ} \\
\text { año escolar }\end{array}$} \\
\hline & $R^{2}$ & $\Delta R^{2}$ & $B$ & $95 \%$ IC de $B$ & $p$ & $R^{2}$ & $\Delta R^{2}$ & $B$ & $95 \%$ IC de $B$ & $p$ \\
\hline $\begin{array}{l}\text { Paso } 1 \\
\text { Vocabulario expresivo }\end{array}$ & 0,12 & 0,12 & 0,34 & $0,28,0,92$ & $\begin{array}{l}<0,001 \\
<0,001\end{array}$ & 0,05 & 0,05 & 0,22 & $0,01,0,59$ & $\begin{array}{l}0,046 \\
0,046\end{array}$ \\
\hline $\begin{array}{l}\text { Paso } 2 \\
\text { CF Cancelación } \\
\text { CF Sustitución } \\
\text { CF Inversión }\end{array}$ & 0,33 & 0,21 & $\begin{array}{l}0,17 \\
0,10 \\
0,34\end{array}$ & $0,31,1,20$ & $\begin{array}{r}<0,001 \\
0,098 \\
0,286 \\
0,001\end{array}$ & 0,09 & 0,04 & $\begin{array}{r}0,12 \\
\text { a } \\
0,15\end{array}$ & & 0,051 \\
\hline $\begin{array}{l}\text { Paso } 3 \\
\text { Correspondencia entre } \\
\text { fonemas y grafemas }\end{array}$ & 0,58 & 0,25 & 0,60 & $0,41,0,69$ & $\begin{array}{l}<0,001 \\
<0,001\end{array}$ & 0,14 & 0,05 & 0,25 & $0,05,0,36$ & 0,019 \\
\hline $\begin{array}{l}\text { Total } R^{2} \\
n\end{array}$ & 0,58 & 104 & & & $<0,001$ & 0,14 & 86 & & & \\
\hline
\end{tabular}

Nota. $\mathrm{CF}=$ Conciencia fonológica. ${ }^{\text {a }} \mathrm{CF}$ Sustitución no fue incluido en el segundo análisis, porque no mostró correlación con la competencia lectora inicial al inicio de $2^{\circ}$ año escolar (ver Tabla 2 ). 
Como se puede ver en la Tabla 3, la inclusión de las tres variables independientes en su conjunto alcanzó un poder predictivo del $14 \%$ de la varianza observada de la competencia lectora inicial de los participantes al inicio del segundo año escolar, $\left.R^{2}=0,14, F(4,81)=52,98, p=0,019\right)$. Las habilidades de vocabulario expresivo, $R^{2}=0,05, F(1,84)=4,09, p=0,046$, y las habilidades de correspondencia entre fonemas y grafemas, $R^{2}=0,05, F(1,81)=3,15, p=0,019$, disminuyeron su potencial predictivo en comparación con los resultados del primer análisis de regresión múltiple, pero aún mostraron un impacto en la competencia lectora inicial al inicio del segundo año escolar. En contraste, las habilidades de conciencia fonológica ya no contribuyeron a explicar la competencia lectora inicial observada al inicio del segundo año escolar, $R^{2}=0,09, F(3,82)=2,69$, $p=0,051$.

\section{Discusión}

A continuación, discutiremos nuestros hallazgos acerca del rol que juegan el vocabulario expresivo, la conciencia fonológica y las relaciones de correspondencia grafema fonema en la competencia lectora que tienen los niños al comenzar el año escolar, así como la variación del poder explicativo de estos precursores un año después.

El hallazgo de que las habilidades de vocabulario expresivo juegan un rol en explicar la variabilidad observada de la competencia lectora inicial en escolares de NSE vulnerable al inicio del primer año escolar es consistente con investigaciones previas tanto en poblaciones angloparlantes como hispanoparlantes (Arriaga et al., 1998; Duff et al., 2015; Duff \& Hulme, 2012; Escobar \& Meneses, 2014; Harlaar et al., 2008; Hart \& Risley, 1995; ; Lesgold \& Perfetti, 1981; Muter et al., 2004; Nation \& Cocksey, 2009; Nation \& Snowling, 2004; NICHD Early Child Care Research Network, 2005; Share, 1995; Wegener et al., 2018). Dentro del contexto latinoamericano, nuestros datos confirman los resultados obtenidos en estudios previos con participantes similares a los incluidos en este estudio, como, por ejemplo, la investigación de Escobar y Meneses (2014). Sin embargo, complementamos la información ofrecida por estos autores sobre el rol de habilidades de vocabulario receptivo con nuevos datos sobre la importancia de las habilidades de vocabulario expresivo sobre la competencia lectora inicial.

Con respecto a las habilidades de conciencia fonológica, nuestros resultados revelan que las subhabilidades de manipulación de fonemas y sílabas, pero no de identificación de rimas, contribuyen a explicar la variabilidad identificada en la competencia lectora inicial al inicio del primer año escolar. Estos resultados son consistentes con hallazgos previos en ortografías superficiales, como el español (CardosoMartins, 1995; Wimmer et al., 1994). La manipulación de unidades fonológicas menores (fonemas aislados o sílabas) es una subhabilidad necesaria para dominar correspondencias biunívocas entre fonemas y grafemas, que predominan en ortografías superficiales. En cambio, en ortografías opacas, como el inglés, las correspondencias entre palabras orales y escritas se basan predominantemente en unidades fonológicas mayores, como la rima. De esta forma, en ortografías opacas subhabilidades como la identificación de rimas sí representan un precursor potente de la competencia lectora inicial (Bryant et al., 1990; Schmalz et al., 2015).

Específicamente en el contexto latinoamericano, nuestros hallazgos confirman la evidencia reportada por Guardia (2014) en un estudio con niños de NSE vulnerable en edad preescolar en Chile, en el cual las habilidades de identificar rimas no aportaron a la explicación de la variabilidad observada en diferentes medidas de competencia lectora inicial evaluadas al final del primer año escolar. Nuestros datos refuerzan este resultado, marcando claramente la diferencia entre la utilidad de emplear tareas de identificación de rimas y de manipulación de fonemas y sílabas para detectar alumnos que tengan un riesgo elevado de presentar dificultades lectoras en poblaciones hispanoparlantes de NSE vulnerable. Mientras Guardia (2014) solamente incluyó una tarea de identificación de rimas, en nuestro estudio medimos diferentes subhabilidades de conciencia fonológica y, de esta forma, está en condiciones de aportar información comparativa sobre el poder explicativo de diferentes subhabilidades de conciencia fonológica sobre la competencia lectora inicial.

El tercer precursor evaluado en este estudio, las habilidades de correspondencia entre fonemas y grafemas, también mostró un poder explicativo de la competencia lectora inicial de niños hispanoparlantes de NSE vulnerable al inicio del primer año escolar. Esta evidencia es consistente con estudios previos, también en el contexto latinoamericano (Byrne \& Fielding-Barnsley, 1989; Ehri, 1998; Escobar \& Meneses, 2014; Foulin, 2005; Hulme et al., 2012; Levin et al., 2006). Así, Escobar y Meneses (2014) reportaron que las habilidades de correspondencia entre fonemas y grafemas, medidas con una tarea de denominación de letras al inicio del primer año escolar, aportaron en la explicación de la variabilidad observada en el rendimiento 
lector evaluado a fines del primer año escolar en niños hispanoparlantes de NSE vulnerable, pero no de NSE medio y alto. Sin embargo, los hallazgos de nuestro estudio están basados en una medida de escritura inventada de palabras y no en una tarea de denominación de letras. De esta forma, nuestro estudio presenta evidencia nueva sobre la utilidad de una tarea de escritura inventada de palabras para identificar alu mnos hispanoparlantes de NSE vulnerable que tengan un riesgo elevado de presentar dificultades en la competencia lectora inicial. Dado que en el estudio de Escobar y Meneses (2014) la tarea de denominación de letras no resultó suficientemente sensible para este fin al evaluar escolares de NSE medio y alto, sería interesante investigar si la tarea de escritura inventada de palabras podría ser una opción alternativa para evaluar las habilidades de correspondencia entre fonemas y grafemas en escolares con un mayor contacto previo con materiales escritos. También una investigación comparativa del poder explicativo de ambas tareas sobre la competencia lectora inicial de niños hispanoparlantes de NSE vulnerables y medios y altos podría ayudar a clarificar este aspecto.

Los hallazgos de que los tres precursores evaluados en nuestro estudio contribuyeron a explicar diferencias individuales en la competencia lectora inicial de niños hispanoparlantes de NSE vulnerable al inicio del primer año escolar dan respuesta a nuestra primera pregunta de investigación. Sin embargo, en relación a nuestra segunda pregunta de investigación, encontramos una disminución del poder explicativo de los tres precursores sobre la competencia lectora inicial evaluada un año más tarde, al inicio del segundo año escolar.

En concordancia con teorías evolutivas del desarrollo lector (e.g., Ehri, 1998; Share, 1995), el rol de las habilidades lingüísticas implicadas en el proceso lector cambia a medida que el lector principiante aumenta su contacto con materiales escritos (Fricke et al., 2016; Tafa \& Manolitsis, 2008; Ziegler et al., 2010). Así, en un primer momento, el desafío consiste en aprender a establecer relaciones entre diferentes unidades de lenguaje oral y escrito (palabras, sílabas y fonemas). Diferentes habilidades de lenguaje oral, como el vocabulario receptivo y expresivo y la conciencia fonológica, claramente juegan un rol primordial para posibilitar el acceso a materiales escritos en esta etapa (Ehri, 1998; Share, 1995). Sin embargo, en ortografías superficiales este desafío es rápidamente superado, gracias a la predominancia de relaciones biunívocas entre unidades de lenguaje oral y escrito. De esta forma, la importancia de las habilidades de lenguaje oral disminuye, como es visible en los resultados de nuestro estudio.

Un segundo desafío, descrito con frecuencia como un obstáculo común específicamente en ortografías superficiales, es la automatización de las correspondencias entre unidades de lenguaje oral y escrito, evidenciada por un aumento en la velocidad lectora (Carrillo \& Alegría, 2009; Defior \& Serrano, 2014; Fricke et al., 2016; Landerl et al., 1997; Morris et al., 2012; Tafa \& Manolitsis, 2008; Wimmer \& Mayringer, 2002; Ziegler et al., 2010). Aquí, medidas que evalúan la velocidad de procesamiento de información auditivo-verbal y visual-ortográfico (e.g., tareas de denominación rápida de letras) parecerían ser prometedoras para identificar alumnos con un riesgo elevado de presentar dificultades en la competencia lectora. Así, por ejemplo, en el estudio de Escobar y Meneses (2014) con escolares hispanoparlantes en Chile la variabilidad en el rendimiento en una tarea de velocidad de denominación medida al inicio del primer año escolar logró explicar diferencias individuales en la lectura de palabras a finales de ese año, aunque solamente con un valor de significancia estadística marginal ( $p=0,049$; ver p. 633 en Escobar \& Meneses, 2014). Además, es probable que la tarea de competencia lectora inicial que usamos en el segundo momento de evaluación, al inicio del segundo año escolar, enfatizara en forma especial la implicancia de la velocidad de procesamiento en relación a las habilidades de lectura, ya que ponderamos tanto la precisión como la velocidad de las respuestas.

\section{Implicaciones Educativas}

Nuestros resultados sugieren implicaciones valiosas para los servicios educativos en contextos de NSE vulnerable. En primer lugar, las habilidades de vocabulario expresivo surgieron como un precursor potente para identificar a niños con un riesgo elevado de presentar dificultades en la competencia lectora inicial al inicio del primer y segundo año escolar. De los tres precursores que investigamos en este estudio, las habilidades de vocabulario expresivo cuentan con la ventaja de que pueden ser estimuladas desde el inicio de la educación preescolar (en muchos países a la edad de 3 años) en lugar de esperar el fracaso. Por lo tanto, recomendamos que centros educativos enfocados en poblaciones hispanoparlantes de NSE vulnerable asignen recursos a fortalecer estas habilidades en las primeras etapas de la educación preescolar.

En segundo lugar, las subhabilidades de conciencia fonológica enfocadas en la manipulación de fonemas y sílabas, y no en la identificación de rimas, fueron capaces de explicar diferencias individuales en la 
competencia lectora inicial a inicios del primer año escolar. Guías para docentes sobre la estimulación de la conciencia fonológica, que a menudo son traducidas y adaptadas de estudios en inglés, destacan la importancia de estimular todo tipo de tarea de conciencia fonológica indistintamente. Sin embargo, resulta imprescindible tomar en cuenta las diferencias entre los sistemas de escritura del inglés y el español. Por lo tanto, sugerimos que en contextos hispanoparlantes el docente centre su atención en la estimulación de habilidades de manipulación de fonemas y sílabas, en vez de actividades con rimas. Sugerimos que estas habilidades deben ser priorizadas al final de la educación preescolar, pero ya no durante el primer año de la escuela primaria, ya que en esta etapa otras habilidades lingüísticas comienzan a tomar un rol más importante para posibilitar el acceso a materiales escritos.

En tercer lugar, las habilidades de correspondencia entre fonemas y grafemas mostraron ser capaces de explicar las diferencias individuales observadas en la competencia lectora inicial al inicio del primer y segundo año escolar. En coherencia con un enfoque preventivo, recomendamos incorporar la enseñanza de esta habilidad de manera sistemática desde el inicio del primer año escolar. También observamos que, además de la tarea de denominación de letras, una tarea de escritura inventada de palabras puede ofrecer información valiosa y, en algunos casos, incluso ser más sensitiva para detectar niños con un riesgo elevado de presentar dificultades en la competencia lectora inicial.

\section{Limitaciones}

Si bien reportamos evidencia innovadora para guiar la identificación de precursores de la competencia lectora inicial en escolares hispanoparlantes de NSE vulnerable, nuestro estudio presenta algunas limitaciones. Primero, la mayoría de los instrumentos de evaluación que usamos fueron estandarizadas con participantes hispanoparlantes residentes en Estados Unidos. Por lo tanto, no contamos con datos sobre la confiabilidad y validez de estos instrumentos para Uruguay.

Segundo, los resultados que reportamos se basan en un subgrupo de escolares con un rendimiento equivalente al percentil 26 o superior en una medida de screening de lectura de grafemas, sílabas y palabras tomada en una muestra más amplia de escolares hispanoparlantes de NSE vulnerable. De esta forma, no queda claro si nuestros resultados también son aplicables a niños con habilidades de lectura severamente descendidas (en y por debajo del percentil 25), en comparación con sus pares. La razón por la que no contamos con estos datos es que priorizamos atender de forma inmediata el riesgo de presentar dificultades lectoras en estos niños por medio de la implementación de una intervención sistematizada intensiva.

Por último, la competencia lectora inicial fue medida por medio de dos tareas diferentes al inicio del primer y segundo año escolar, respectivamente. Mientras esta decisión metodológica nos posibilitó tomar en cuenta las exigencias específicas de diferentes momentos evolutivos del desarrollo lector (e.g., la automatización de procesos léxicos implicados en la lectura), también dificultó la comparación del poder explicativo de los tres precursores evaluados en nuestro estudio sobre la competencia lectora inicial en dos etapas escolares diferentes. De esta forma, en futuras investigaciones sería conveniente evaluar diferentes aspectos de la competencia lectora inicial en diferentes momentos evolutivos.

\section{Conclusiones}

El presente estudio tuvo como objetivo contribuir hacia la construcción de una base empírica para la identificación de precursores de la competencia lectora inicial en escolares hispanoparlantes de NSE vulnerable. Obtuvimos hallazgos que sugieren precaución al trasladar implicaciones educativas derivadas de estudios realizados con escolares angloparlantes. Nuestros datos confirman la importancia de considerar los mismos precursores que se observan en la literatura: vocabulario, conciencia fonológica y correspondencia entre fonemas y grafemas, pero también muestran que tienen diferente poder explicativo, y que el papel de

la conciencia fonológica desaparece un año después de la exposición a la enseñanza explícita de la competencia lectora.

\section{Referencias}

Administración Nacional de Educación Pública (2013). Programa de educación inicial y primaria (3ª ed.). Montevideo, Uruguay: Autor, Consejo de Educación Secundaria.

Arriaga, R. I., Fenson, L., Cronan, T. \& Pethick, S. J. (1998). Scores on the MacArthur Communicative Development Inventory of children from low and middle-income families. Applied Psycholinguistics, 19, 209-223. https://doi.org/10.1017/S0142716400010043 
Boyes, M. E., Leitao, S., Claessen, M., Badcock, N. A. \& Nayton, M. (2016). Why are reading difficulties associated with mental health problems? Dyslexia, 22, 263-266. https://doi.org/10.1002/dys.1531

Bridges, M. S. \& Catts, H. W. (2011). The use of a dynamic screening of phonological awareness to predict risk for reading disabilities in kindergarten children. Journal of Learning Disabilities, 44, 330-338. https://doi.org/10.1177/0022219411407863

Bryant, P. E., MacLean, M., Bradley, L. L. \& Crossland, J. (1990). Rhyme and alliteration, phoneme detection, and learning to read. Developmental Psychology, 26, 429-438. https://doi.org/10.1037/0012-1649.26.3.429

Byrne, B. \& Fielding-Barnsley, R. (1989). Phonemic awareness and letter knowledge in the child's acquisition of the alphabetic principle. Journal of Educational Psychology, 81, 313-321. https://doi.org/10.1037/0022-0663.81.3.313

Caravolas, M., Lervåg, A., Mousikou, P., Efrim, C., Litavský, M., Onochie-Quintanilla, E. ... Hulme, C. (2012). Common patterns of prediction of literacy development in different alphabetic orthographies. Psychological Science, 23, 678-686. https://doi.org/10.1177/0956797611434536

Caravolas, M., Volín, J. \& Hulme, C. (2005). Phoneme awareness is a key component of alphabetic literacy skills in consistent and inconsistent orthographies: Evidence from Czech and English children. Journal of Experimental Child Psychology, 92, 107-139. https://doi.org/10.1016/j.jecp.2005.04.003

Cardoso-Martins, C. (1995). Sensitivity to rhymes, syllables, and phonemes in literacy acquisition in Portuguese. Reading Research Quarterly, 30, 808-828. https://doi.org/10.2307/748199

Carrillo, M. \& Alegría, J. (2009). Mecanismos de identificación de palabras en niños disléxicos en español: ¿existen subtipos? Ciencias Psicológicas, 3, 135-152. https://doi.org/10.22235/cp.v3i2.146

Carta, J. J., Greenwood, C. R., Atwater, J., McConnell, S. R., Goldstein, H. \& Kaminski, R. A. (2014). Identifying preschool children for higher tiers of language and early literacy instruction within a response to intervention framework. Journal of Early Intervention, 36, 281-291. https://doi.org/10.1177/1053815115579937

Cuadro, A., Barg, G., Navarrete, I. \& Suero, M. (2008). Evaluación de las competencias cognitivas y sociales de niños que han estado en situación de calle. Ciencias Psicológicas, 2, 143-152. https://doi.org/10.22235/cp.v0i2.647

Cuadro, A., Marín, J., Carrillo, M., Trías, D., Ponce de León, P. \& Costa, D. (2005). Test de Eficacia Lectora TECLE. Montevideo, Uruguay: Prensa Médica Latinoamericana.

Cunningham, P. M. \& Cunningham, J. W. (1992). Making words: Enhancing the invented spelling-decoding connection. The Reading Teacher, 46, 106-115. Extraído de https://www.jstor.org/stable/pdf/20201026.pdf

Defior, S. \& Serrano, F. (2014). Diachronic and synchronic aspects of Spanish: The relationship with literacy acquisition. Estudios de Psicología, 35, 450-475. https://doi.org/10.1080/02109395.2014.974422

Dijkstra, T. \& van Heuven, W. J. B. (2002). The architecture of the bilingual word recognition system: From identification to decision. Bilingualism: Language and Cognition, 5, 175-197. https://doi.org/10.1017/S1366728902003012

Duff, F. J. \& Hulme, C. (2012). The role of children's phonological and semantic knowledge in learning to read words. Scientific Studies of Reading, 16, 504-525. https://doi.org/10.1080/10888438.2011.598199

Duff, F. J., Reen, G., Plunkett, K. \& Nation, K. (2015). Do infant vocabulary skills predict school-age language and literacy outcomes? The Journal of Child Psychology and Psychiatry, 56, 848-856. https://doi.org/10.1111/jcpp.12378

Ehri, L. C. (1998). Grapheme-phoneme knowledge is essential to learning to read words in English. En J. L. Metsala \& L. C. Ehri (Eds.), Word recognition in beginning literacy (pp. 3-40). Mahwah, NJ: Lawrence Erlbaum.

Escobar, J. P. \& Meneses, A. (2014) Initial reading predictors in Spanish according to SES: Is semi-transparency sufficient to explain performance? Estudios de Psicología, 35, 625-635. https://doi.org/10.1080/02109395.2014.965458

Foulin, J. N. (2005). Why is letter-name knowledge such a good predictor of learning to read? Reading and Writing, 18, 129-155. https://doi.org/10.1007/s11145-004-5892-2

Fricke, S., Szczerbinski, M., Fox-Boyer, A. \& Stackhouse, J. (2016). Preschool predictors of early literacy acquisition in German-speaking children. Reading Research Quarterly, 51, 29-53. https://doi.org/10.1002/rrq.116

Friedmann, N. \& Coltheart, M. (2018). Types of developmental dyslexia. En A. Bar-On \& D. Ravid (Eds.), Handbook of communication disorders: Theoretical, empirical, and applied linguistic perspectives (pp. 721-751). Berlin, Alemania: De Gruyter Mouton.

Gentry, J. R. (2000). A retrospective on invented spelling and a look forward. The Reading Teacher, 54, 318-332. Extraído de https://www.jstor.org/stable/20204910?seq=1\#metadata_info_tab_contents.

Georgiou, G. K., Parrila, R. \& Papadopoulos, T. C. (2008). Predictors of word decoding and reading fluency across languages varying in orthographic consistency. Journal of Educational Psychology, 100, 566-580. https://doi.org/10.1037/0022-0663.100.3.566

Geva, E. \& Siegel, L. S. (2000). Orthographic and cognitive factors in the concurrent development of basic reading skills in two languages. Reading and Writing, 12, 1-30. https://doi.org/10.1023/A:1008017710115

Griffin, E. A. \& Morrison, F. J. (1997). The unique contribution of home literacy environment to differences in early literacy skills. Early Child Development and Care, 127, 233-243. https://doi.org/10.1080/0300443971270119

Guardia, P. (2014). Is consonant rhyme important when learning to read in Spanish? Estudios de Psicología, 35, 567583. https://doi.org/10.1080/02109395.2014.965459

Harlaar, N., Hayiou-Thomas, M. E., Dale, P. S. \& Plomin, R. (2008). Why do preschool language abilities correlate with later reading? A twin study. Journal of Speech, Language, and Hearing Research, 51, 688-705. https://doi.org/10.1044/1092-4388(2008/049)

Hart, B. \& Risley, T. R. (1995). Meaningful differences in the everyday experience of young American children. Baltimore, MD: Paul H. Brookes.

Hoff, E. (2013). Interpreting the early language trajectories of children from low-SES and language minority homes: Implications for closing achievement gaps. Developmental Psychology, 49, 4-14. https://doi.org/10.1037/a0027238

Hulme, C., Bowyer-Crane, C., Carroll, J. M., Duff, F. J. \& Snowling, M. J. (2012). The causal role of phoneme awareness and lettersound knowledge in learning to read: Combining intervention studies with mediation analyses. Psychological Science, 23, 572-577. https://doi.org/10.1177/0956797611435921

Huttenlocher, J., Waterfall, H., Vasilyeva, M., Vevea, J. \& Hedges, L. V. (2010). Sources of variability in children's language growth. Cognitive Psychology, 61, 343-365. https://doi.org/10.1016/j.cogpsych.2010.08.002

Kavale, K. A. (2005). Identifying specific learning disability: Is responsiveness to intervention the answer? Journal of Learning Disabilities, 38, 553-562. https://doi.org/10.1177/00222194050380061201

Lam, E. A. \& McMaster, K. L. (2014). Predictors of responsiveness to early literacy intervention: A 10-year update. Learning Disability Quarterly, 37, 134-147. https://doi.org/10.1177/0731948714529772 
Landerl, K., Wimmer, H. \& Frith, U. (1997). The impact of orthographic consistency on dyslexia: A German-English comparison. Cognition, 63, 315-334. https://doi.org/10.1016/S0010-0277(97)00005-X

Lesgold, A. M. \& Perfetti, C. A. (Eds.) (1981). Interactive processes in reading. London, Reino Unido: Routledge.

Levin, I., Shatil-Carmon, S. \& Asif-Rave, O. (2006). Learning of letter names and sounds and their contribution to word recognition. Journal of Experimental Child Psychology, 93, 139-165. https://doi.org/10.1016/j.jecp.2005.08.002

Lombardino, L. J., Bedford, T., Fortier, C., Carter, J. \& Brandi, J. (1997). Invented spelling: Developmental patterns in kindergarten children and guidelines for early literacy intervention. Language, Speech, and Hearing Services in Schools, 28, 333-343. https://doi.org/10.1044/0161-1461.2804.333

Mayer, A. \& Motsch, H. -J. (2015). Efficacy of a classroom integrated intervention of phonological awareness and word recognition in "double-deficit children" learning a regular orthography. Journal of Education and Learning, 4(3), 88-111. https://doi.org/10.5539/jel.v4n3p88

Morris, R. D., Lovett, M. W., Wolf, M., Sevcik, R. A., Steinbach, K. A., Frijters, J. C. \& Shapiro, M. B. (2012). Multiple-component remediation for developmental reading disabilities: IQ, socioeconomic status, and race as factors in remedial outcome. Journal of Learning Disabilities, 45(2), 99-127. https://doi.org/10.1177/0022219409355472

Muñoz-Sandoval, A. F., Woodcock, R. W., McGrew, K. S. \& Mather, N. (2005). Batería III Woodcock-Muñoz: pruebas de habilidades cognitivas. Itasca, IL: Riverside.

Muter, V., Hulme, C., Snowling, M. J. \& Stevenson, J. (2004). Phonemes, rimes, vocabulary, and grammatical skills as foundations of early reading development: Evidence from a longitudinal study. Developmental Psychology, 40, 665-681. https://doi.org/10.1037/0012-1649.40.5.665

Nation, K. \& Cocksey, J. (2009). The relationship between knowing a word and reading it aloud in children's word reading development. Journal of Experimental Child Psychology, 103, 296-308. https://doi.org/10.1016/j.jecp.2009.03.004

Nation, K. \& Snowling, M. J. (2004). Beyond phonological skills: Broader language skills contribute to the development of reading. Journal of Research in Reading, 27, 342-356. https://doi.org/10.1111/j.1467-9817.2004.00238.x

NICHD Early Child Care Research Network (Ed.) (2005). Child care and child development: Results from the NICHD study of early child care and youth development. New York, NY: Guilford Press.

Ouellette, G. \& Sénéchal, M. (2008). Pathways to literacy: A study of invented spelling and its role in learning to read. Child Development, 79, 899-913. https://doi.org/10.1111/j.1467-8624.2008.01166.x

Ouellette, G. \& Sénéchal, M. (2017). Invented spelling in kindergarten as a predictor of reading and spelling in grade 1: A new pathway to literacy, or just the same road, less known? Developmental Psychology, 53, 77-88. https://doi.org/10.1037/dev0000179

Piasta, S. B. \& Wagner, R. K. (2010). Developing early literacy skills: A meta-analysis of alphabet learning and instruction. Reading Research Quarterly, 45, 8-38. https://doi.org/10.1598/RRQ.45.1.2

Reschly, D. J. (2005). Learning disabilities identification: Primary intervention, secondary intervention, and then what? Journal of Learning Disabilities, 38, 510-515. https://doi.org/10.1177/00222194050380060601

Roth, F. P., Speece, D. L. \& Cooper, D. H. (2002). A longitudinal analysis of the connection between oral language and early reading. The Journal of Educational Research, 95, 259-272. https://doi.org/10.1080/00220670209596600

Rumelhart, D. E. (1975, Julio/1977). Toward an interactive model of reading. En S. Dornič (Ed.), Attention and performance VI. Proceedings of the Sixth International Symposium on Attention and Performance, Stockholm, Suecia (Vol. 6, pp. 573-603). Hillsdale, NJ: Lawrence Erlbaum.

Rumelhart, D. E. (1994). Toward an interactive model of reading. En R. B. Ruddell, M. R. Ruddell \& H. Singer (Eds.), Theoretical models and processes of reading ( $4^{\mathrm{a}} \mathrm{ed} ., \mathrm{pp}$. 864-894). Newark, DE: International Reading Association.

Scarborough, H. S. (2009). Connecting early language and literacy to later reading (dis)abilities: Evidence, theory, and practice. En F. Fletcher-Campbell, J. Soler \& G. Reid (Eds). Approaching difficulties in literacy development: Assessment, pedagogy, and programmes (pp. 23-39). London, Reino Unido: SAGE.

Schmalz, X., Marinus, E., Coltheart, M. \& Castles, A. (2015). Getting to the bottom of orthographic depth. Psychonomic Bulletin \& Review, 22, 1614-1629. https://doi.org/10.3758/s13423-015-0835-2

Schrank, F. A., McGrew, K. S., Ruef, M. L., Alvarado, C. G., Muñoz-Sandoval, A. F. \& Woodcock, R. W. (2005). Batería III WoodcockMuñoz: Overview and technical supplement (Assessment Service Bulletin N ${ }^{\circ}$ 1). Rolling Meadows, IL: Riverside.

Share, D. L. (1995). Phonological recoding and self-teaching: Sine qua non of reading acquisition. Cognition, 55, 151-218. https://doi.org/10.1016/0010-0277(94)00645-2

Share, D. L. (2008). On the Anglocentricities of current reading research and practice: The perils of overreliance on an "outlier" orthography. Psychological Bulletin, 134, 584-615. https://doi.org/10.1037/0033-2909.134.4.584

Stage, S. A., Abbott, R. D., Jenkins, J. R. \& Berninger, V. W. (2003). Predicting response to early reading intervention from verbal IQ, reading-related language abilities, attention ratings, and verbal IQ-word reading discrepancy: Failure to validate discrepancy method. Journal of Learning Disabilities, 36, 24-33. https://doi.org/10.1177/00222194030360010401

Strijkers, K., Bertrand, D. \& Grainger, J. (2015). Seeing the same words differently: The time course of automaticity and top-down intention in reading. Journal of Cognitive Neuroscience, 27, 1542-1551. https://doi.org/10.1162/jocn_a_00797

Tafa, E. \& Manolitsis, G. (2008). A longitudinal literacy profile of Greek precocious readers. Reading Research Quarterly, 43, $165-185$. https://doi.org/10.1598/RRQ.43.2.4

Tunmer, W. \& Greaney, K. (2010). Defining dyslexia. Journal of Learning Disabilities, 43, 229-243. https://doi.org/10.1177/0022219409345009

Uruguay, Instituto Nacional de Estadística (2012). Indice de nivel socioeconómico (INSE). Montevideo, Uruguay: Autor. Extraído de http://www.gruporadar.com.uy/01/wp-content/uploads/2012/04/Informe-Nuevo-INSE-2011.pdf

Vellutino, F. R., Scanlon, D. M., Zhang, H. \& Schatschneider, C. (2008). Using response to kindergarten and first grade intervention to identify children at-risk for long-term reading difficulties. Reading and Writing, 21, 437-480. https://doi.org/10.1007/s11145-007-9098-2

Wegener, S., Wang, H. C., de Lissa, P., Robidoux, S., Nation, K. \& Castles, A. (2018). Children reading spoken words: Interactions between vocabulary and orthographic expectancy. Developmental Science, 21(3), artículo e12577. https://doi.org/10.1111/desc.12577

Wimmer, H., Landerl, K., Linortner, R. \& Hummer, P. (1991). The relationship of phonemic awareness to reading acquisition: More consequence than precondition but still important. Cognition, 40, 219-249. https://doi.org/10.1016/0010-0277(91)90026-Z

Wimmer, H., Landerl, K. \& Schneider, W. (1994). The role of rhyme awareness in learning to read a regular orthography. British Journal of Developmental Psychology, 12, 469-484. https://doi.org/10.1111/j.2044-835X.1994.tb00648.x

Wimmer, H. \& Mayringer, H. (2002). Dysfluent reading in the absence of spelling difficulties: A specific disability in regular orthographies. Journal of Educational Psychology, 94, 272-277. https://doi.org/10.1037/0022-0663.94.2.272 
Ziegler, J. C., Bertrand, D., Tóth, D., Csépe, V., Reis, A., Faísca, L. ... Blomert, L. (2010). Orthographic depth and its impact on universal predictors of reading: A cross-language investigation. Psychological Science, 21, 551-559. https://doi.org/10.1177/0956797610363406

Ziegler, J. C. \& Ferrand, L. (1998). Orthography shapes the perception of speech: The consistency effect in auditory word recognition. Psychonomic Bulletin \& Review, 5, 683-689. https://doi.org/10.3758/BF03208845

Ziegler, J. C. \& Goswami, U. (2006). Becoming literate in different languages: Similar problems, different solutions. Developmental Science, 9, 429-436. https://doi.org/10.1111/j.1467-7687.2006.00509.x

Fecha de recepción: Abril de 2018.

Fecha de aceptación: Junio de 2019. 